\title{
Serum Levels of Insulin Like Growth Factor-1(IGF-1) in Patients with Seborrheic Dermatitis
}

\author{
M.Z.Kenawy ${ }^{1}$, N.W.Mikhael ${ }^{1}$, A.A.El-fallah ${ }^{2}$ and R.A.Shehata ${ }^{1}$
}

Dermatology, Venereology and Andrology, Clinical and Chemical Pathology Dept., Faculty of Medicine, Benha Univ., Benha, Egypt

E-Mail:roon4tone@gmail.com

\begin{abstract}
Seborrheic dermatitis (SD) is a chronic inflammatory skin disease affecting sebaceous areas of the face, scalp, upper chest and back .Five factore are believed to play a key role in the development of SD lesions: excess sebum production, malassezia colonies over areas covered with lipids, lipase secreted by malassezia, release of inflammatory cytokines into the skin, keratinocyte proliferation and differentiation. Evaluation of serum levels of Insulin Like Growth Factor-1(IGF-1) in seborrheic dermatitis patients. Serum IGF-1 concentrations were measured in 50 patients with SD and 30 healthy controls using enzyme-linked immunosorbent assay (ELISA). Serum levels of IGF-1 in patients with SD were found to be higher than in healthy controls $(\mathrm{P}=0.045)$. Increased serum IGF-1 levels probably reflect the role of IGF-1 in the pathogenesis of SD.
\end{abstract}

Keywords: Seborrheic dermatitis, IGF-1, ELISA.

\section{Introduction}

Seborrheic dermatitis (SD) is a common chronic relapsing inflammatory skin disease affecting sebaceous areas of the scalp, hair line, eyebrows, nasolabial folds, glabella, ears, upper chest and back and characterized by folliculo-centric salmon-colored papules and plaques with a fine white scale, and a yellowish crust often described as a greasy scale crust [1].

Multiple factors are involved in the pathogenesis of SD, including: lipids secreted from sebaceous glands onto the skin surface, Malassezia colonies over those areas covered with lipids, lipase secreted by Malassezia resulting in generation of free fatty acids and lipid peroxides that activate inflammatory cytokines such as IL-1 $\alpha$, IL-1 $\beta$, IL-2, IL-4, IL-6, IL-8, IL-10, IL-12 and $\mathrm{TNF}-\alpha$, leading to keratinocyte proliferation and differentiationan [2].

Insulin like growth factor-1 (IGF-1) is a peptide hormone similar in the molecular structure to insulin and encoded by IGF-1 gene. IGF-1 circulate in the blood stream, about $99 \%$ of the total IGF-1 circulate in the blood binding to a high affinity binding proteins called (IGFBPs) [3].

IGF-1 is synthesized in the skin mainly by the dermal fibroblasts. It stimulates sebocyte proliferation and differentiation resulting in stimulating the lipogenesis. It also promotes the clonal proliferation of keratinocytes and stimulates $5 \alpha$-reductase, adrenal and androgen synthesis, androgen receptor signal transduction leading to increase sebum secretion [4].

The increase of sebum production often relates with the inflammatory skin diseases. The excess sebum production is the primary event, followed by hyperkeratinization of pilosebaceous duct and perifollicular inflammation and the release of inflammatory cytokines [5].

So, IGF-1 may play a role in the pathogenesis of seborrheic dermatitis. The aim of this study is to asses the serum level of IGF-1 in patients with seborrheic dermatitis.

\section{Material and methods}

This case control study included 50 patients with seborrheic dermatitis and 30 healthy age and sex matched controls attending to the dermatology outpatient clinic at Benha University Hospital, Benha, Egypt from December 2019 to July 2020. Participants gave their informed written consent before enrolment and the study was approved by the Research Ethics Committee in Faculty of Medicine, Benha University.

\subsection{History taking}

Patients' demographic data were recorded: name, age,sex and occupation. A detailed history was taken from patients regarding onset, course, duration of SD. Also we asked about medical problems including any systemic or skin diseases, drug intake and family history.

\subsection{Clinical examination}

Full general examination was done to exclude associated systemic diseases and other inflammatory skin diseases, Detailed dermatological examination; for detection of different SD lesions by examination of the scalp, nasolabial folds, ears, eyebrows, chest and back and finally evaluating the severity according to SEDASI score [6].

\section{3 blood samples}

Three mls of venous blood samples were collected from each participant under complete aseptic conditions and put in a serum separating tube and left for 30 minutes till clotting, then samples were centrifuged at $2000 \mathrm{~g}$ for 10 minutes.

The separated serum were aliquoted and stored at ($30^{\circ}$ c) till assay.

Determination of serum level of Insulin like growth factor-1 were done using enzyme linked Immunosorbent Assay (ELISA) Kits provided by Shanghai Sunred Biological Technology Company, China, according to the manufacturer's instructions. 


\subsection{Statistical analysis}

The collected data were tabulated and analyzed using SPSS vs.25.(IBM, Armonk, New York, United states).

\section{Results and discussion} 3.1 Laboratory results
The current study was carried out on 50 patients with SD. The control group included 30 healthy subjects.

There was a statistically significant higher value in the mean serum level of IGF-1 in patients with SD than in controls Table (1).

Also median IGF-1 was higher in severe cases compared to mild and moderate cases Table (2).

Table (1) Comparison of serum IGF-1 concentration between studied groups.

\begin{tabular}{llll}
\hline & Seborrheic dermatitis & Control & P value \\
\hline & $\mathbf{N}=\mathbf{5 0}$ & $\mathbf{N}=\mathbf{3 0}$ & \\
\hline mean \pm SD & mean \pm SD & \\
\hline IGF-1 $(\mathbf{n g} / \mathbf{m L})$ & $1.33(0.75-15.32)$ & $1.20(0.1-7.95)$ & 0.045 \\
\hline
\end{tabular}

Table (2) Serum IGF-1 levels as regard disease severity.

\begin{tabular}{ccccc}
\hline & Mild & Moderate & Severe & P value \\
\hline & $\mathbf{N}=\mathbf{2 1}$ & $\mathbf{N}=\mathbf{1 8}$ & $\mathbf{N}=\mathbf{1 1}$ & \\
\hline mean \pm SD & mean \pm SD & mean \pm SD & \\
\hline IGF-1 $(\mathbf{n g} / \mathbf{m L})$ & $1.32(0.75-2.98)$ & $1.33(0.91-3.49)$ & $3.31(1.21-15.32)$ & 0.005 \\
\hline
\end{tabular}

\section{Discussion}

Seborrheic dermatitis is a common chronic relapsing inflammatory skin disease presents as a red scaly rash, affecting sebaceous areas of the face, scalp, upper chest and back with high sebum secretion, malassezia colonies and inflammatory cytokines playing the role in its pathogenesis [7].

Insulin like growth factor-1(IGF-1) is a peptide hormone similar in the molecular structure to insulin, produced mainly by the liver under GH stimulus, also secreted in the skin mainly by dermal fibroblast and promotes the clonal proliferation of keratinocytes and stimulates $5 \alpha$-reductase, adrenal and androgen synthesis and lipogenesis leading to increase sebum secretion which strongly indicates the potential of its use as a biomarker of SD onset and progression $[3,4]$.

The aim of this work was to estimate serum level of IGF-1 to assess its role in seborrheic dermatitis.

This was done through evaluation of 50 SD patients. The patients were subdivided into three groups according to the severity of their disease; mild group $(n=21)$, moderate $(n=18)$ and severe group $(n=11) .30$ healthy individuals of matched age and sex as a control group .

According to results of the current study, there was a statistically significant increase $(\mathrm{p}=0.045)$ in serum IGF-1 level among cases compared to control group. Also median IGF-1 was significantly higher in severe cases compared to mild and moderate cases.

To the best of our knowledge, no published studies were found to asses the level of IGF-1 in seborrheic dermatitis patients.

The results of the present study were consistent with the results of the study conducted by Kim et al. in 2017; which found that IGF-1 increases the expression of inflammatory biomarkers and sebum production in cultured sebocytes [8] Also different studies have reported increase in serum IGF-1 levels in other inflammatory diseases. For example, studies reported increase in serum level of IGF-1 in acne patients than in controls $[9,10,11,12,13,14]$.

Other studies also reported the involvement of IGF-1 in the pathogenesis of psoriasis $[15,16]$.

Although the role of IGF-1 in inflammation is appreciated, its involvement in the pathogenesis of SD has not been well elucidated. However, a line of evidence suggests the pathogenic role of IGF-1 in SD. For example, IGF-1 is found to increase sebum production and inflammatory cytokines as IL-1 $\beta$, IL-6, IL-8, and TNF- $\alpha$ which play an important role in many stages in the pathogenesis of SD [2].

Consistent with this idea, it is not surprising that this study found increased serum levels of IGF-1 in SD, so it is considered to be a promising target in treating this disease.

\section{Conclusion}

Our results showed the contribution of increased IGF-1 in the development of seborrheic dermatitis.

\section{References}

[1] L.C.Paulino. New perspectives on dandruff and seborrheic dermatitis: lessons we learned from bacterial and fungal skin microbiota. European Journal of Dermatology, Vol.27(1),PP.4-7,2017.

[2] J.A.Adalsteinsson, S.Kaushik, S.Muzumdar. An update on the microbiology, immunology and genetics of seborrheic dermatitis. Experimental Dermatology, Vol.29(5), PP.481-489,2020.

[3] B.C.Nindl. Insulin-like growth factor-I as a candidate metabolic biomarker: military relevance and future directions for measurement. Journal of Diabetes Science and Technology, Vol.3(2), PP.371376,2009.

[4] B.C.Melnik, G.Schmitz. Role of insulin, insulin-like growth factor-1, hyperglycaemic food and milk 
consumption in the pathogenesis of acne vulgaris. Experimental dermatology, Vol.18(10), PP.833841.2009.

[5] B.Dreno, H.P.M.Gollnick, S.Kang. to Improve Outcomes in Acne, Understanding innate immunity and inflammation in acne: implications for management. Journal of the European Academy of Dermatology and Venereology, Vol. 29, PP.311,2015 .

[6] G.Micali, F.Lacarrubba, F.Dall'Oglio. A new proposed severity score for seborrheic dermatitis of the face: SEborrheic Dermatitis Area and Severity Index (SEDASI): 4600. Journal of the American Academy of Dermatology, Vol.76,PP.(6),2017.

[7] S.Y.Kim, S.H.Kim, S.N.Kim. Isolation and identification of Malassezia species from Chinese and Korean patients with seborrheic dermatitis and in vitro studies on their bioactivity on sebaceous lipids and IL-8 production. Mycoses.Vol.59(5), PP.274-280,2016.

[8] H.Kim, S.Y.Moon, M.Y.Sohn. Insulin-like growth factor-1 increases the expression of inflammatory biomarkers and sebum production in cultured sebocytes. Annals of dermatology.Vol.29(1), PP.20$25,2017$.

[9] S.El-Tahlawi, N.Ezzat Mohammad, A.Mohamed ElAmir. Survivin and insulin-like growth factor-I: potential role in the pathogenesis of acne and postacne scar. Scars, burns \& healing.Vol.5, p.2059513118818031,2019.

[10] H.A.Assaf, W.M.Abdel-Maged, B.E.Elsadek. Survivin as a novel biomarker in the pathogenesis of acne vulgaris and its correlation to insulin-like growth factor-I. Disease markers, 2016.

[11] M. \Polat, \ M.Ekşioğlu. Serum Growth Hormone and Insulin-Like Growth Factor-1 Levels in Women with Postadolescent Acne. TURKDERM-Archieves of The Turkish Dermatology and Venerology,Vol.44(2), PP.69-72,2010.

[12] N.Hunter, O.Shaker. Diet and body mass index, do they have a role in acne vulgaris? A study using serum leptin and serum lnsulin like growth factor-1. Egypt J Derm Androl, Vol.9(2), PP.73-80, 2009.

[13] M.Cappel, D.Mauger, D.Thiboutot. Correlation between serum levels of insulin-like growth factor 1, dehydroepiandrosterone sulfate, and dihydrotestosterone and acne lesion counts in adult women. Archives of dermatology, Vol.141(3), PP.333-338, 2005.

[14] O.Isard, A.C.Knol, M.F.Ariès. Propionibacterium acnes activates the IGF-1/IGF-1R system in the epidermis and induces keratinocyte proliferation. Journal of investigative dermatology, Vol.131(1), PP.59-66, 2011.

[15] H.Miura, S.Sano, M.Higashiyama. Involvement of insulin-like growth factor-I in psoriasis as a paracrine growth factor: dermal fibroblasts play a regulatory role in developing psoriatic lesions. Archives of dermatological research, Vol.292(12), PP.590-597, 2000.

[16] C.J.Wraight, P.J.White, S.C.McKean. Reversal of epidermal hyperproliferation in psoriasis by insulinlike growth factor I receptor antisense oligonucleotides. Nature biotechnology, Vol.18(5), PP.521-526, 2000. 